\title{
VECTOR FIELDS AND TRANSFERS
}

\section{JAMES C. BECKER AND DANIEL HENRY GOTTLIEB}

For a smooth fibre bundle $F \stackrel{i}{\longrightarrow} E \stackrel{p}{\longrightarrow} B$ where $F$ is a compact manifold with or without boundary, a vertical vector field $V$ gives rise to a transfer $\tau_{V}$ as an $S$-map. Our goal is to show these transfers satisfy an equation analogous to one that the index of vector fields satisfy. This equation gives results involving equivariant vector fields as well as a characterization of those transfers defined by vector fields in terms of the ordinary Euler-Poincare transfers.

\section{Introduction}

Let $F \stackrel{i}{\longrightarrow} E \stackrel{p}{\longrightarrow} B$ be a smooth fibre bundle with $F$ a compact manifold with boundary $\partial F$ and $B$ a closed manifold. Let $V$ be a vector field defined on an open set of $E$ which is tangent to the fibres. We assume that $V$ has no zeros on $\partial E=\dot{E}$. We will call such vector fields vertical vector fields.

We define an $S$-map $\tau_{V}: B^{+} \rightarrow E^{+}$associated with $V$. This transfer $\tau_{V}$ has the usual properties:

a) If $V$ is homotopic to a vertical vector field $V^{\prime}$ by a homotopy of vertical vector fields, so in particular no zeros appear on $\partial E$, then $\tau_{V^{\prime}}$ is homotopic to $\tau_{V}$.

b) $\tau_{V}^{*}\left(p^{*} \alpha \cup \beta\right)=\alpha \cup \tau_{V}^{*}(\beta)$ for cohomology theories $h^{*}$ with cup products.

c) For ordinary homology or cohomology, $p_{*} \circ \tau_{V_{*}}$ and $\tau_{V}^{*} \circ p^{*}$ is multiplication by the index of $V$ restricted to a fibre $F$, denoted $\operatorname{Ind}(V \mid F)$.

The main result of this paper is the analogue of the following equation for indices of vector fields, which we call the Law of Vector Fields.

$$
\text { Ind } V+\operatorname{Ind} \partial_{-} V=\chi(M)
$$




\section{BECKER - GOTTLIEB}

Here $M$ is a compact manifold and $\partial_{-} V$ is the vector field $V$ restricted to $\partial_{-} M$, that open set of the boundary where $V$ points inward and then projected down tangent to the boundary.

The analogous equation for transfers is

$$
\tau_{V}+i \circ \tau_{\partial_{-} V}=\tau
$$

where $\tau_{\partial_{-}}$is a transfer $B^{+} \rightarrow \dot{E}^{+}$based on $\partial_{-} V$ and $i: \dot{E} \hookrightarrow E$ is the inclusion. The $\tau$ on the right hand side is the usual EulerPoincare transfer of the fibre bundle, [1] or [2].

We use these transfers in Section 9 to show for a fibre bundle $F \stackrel{i}{\longrightarrow} E \stackrel{p}{\longrightarrow} B$ where $V$ is a vertical vector field, that

$$
0=\operatorname{Ind}(V \mid F) \omega_{*}:\{X, \Omega B\} \rightarrow\{X, F\}
$$

is trivial. Here we assume that $X$ is a finite complex, $\Omega B \stackrel{\omega}{\longrightarrow} F$ is the transgression map induced by the fibre bundle, $\{X, Y\}$ denotes the group of stable homotopy classes from $X$ to $Y$.

Vertical vector fields can be constructed from equivariant vector fields on a manifold with a group action. Thus if $V$ is a $G$-vector field on a manifold $M$, then the trace of the action $(G, M)$, denoted by $\operatorname{tr}(G, M)$ and defined in [3], must divide Ind $V$. In symbols,

$$
\operatorname{tr}(G, M) \mid \operatorname{Ind}(V)
$$

Relation (4) and its numerous consequences are examined in Section 8.

This paper employs the techniques first published in [1]. The techniques in that paper gave rise to a transfer associated to vector fields which point outward on the boundary. Rudzinski, [10], examined the situation when the vector field was equivariant, and defined transfers in that case. When the zeros of the equivariant vector field are isolated, the transfer reduces to that of the covering bundle given by the equivariant zeros. 


\section{BECKER - GOTTLIEB}

In this paper we consider non-equivariant vector fields. In our approach we come closer to the way Dold constructs his fixed point transfer, [7]. Dold uses fibre preserving "compactly fixed" maps defined on open sets of the total spaces. Since Dold is assuming ENR's, his maps can be converted to vertical vector fields by a construction like $V(e)=e-f(e)$. On the other hand, the vertical vector fields give rise to fibre preserving maps.

So Dold's fibre preserving map approach and our vertical vector field approach to transfers seem to be covering the same ground. Dold's approach can be applied to more general situations than ours. We need smooth manifolds so that we can talk about vector fields and tangent bundles. On the other hand, we define the transfer more directly than in Dold's paper.

Dold gives in $[4,5,6,7]$, and a survey [8], a treatment of the fixed point index which steadily generalizes to give the fixed point transfer. In this paper, we define the vector field index by means of an $S$-map and steadily generalize until we get the vector field transfer, so we follow Dold's point of view.

The fixed point index and the vector field index have an enigmatic relationship. Each can be defined in terms of the other (except for the greater generality of the fixed point index setting). On the other hand, some principal properties appear different. Maps compose, and the fact that the fixed point index is invariant under the change of order of composition is a fundamental property which does not have an obvious analogue for vector fields. Conversely, the immensely useful fact that $\operatorname{Ind}(-V)=(-1)^{n} \operatorname{Ind}(V)$ does not have an obvious analogue for the fixed point index.

\section{The index of a global vector field}

\subsection{Definition of $\tau_{V}$}

Let $F$ be a compact manifold with boundary $\dot{F}$. Let $V$ be a vector field so that $V \mid \dot{F}$ has no zeros. Then we define an element $\left\{\tau_{V}\right\}$ in the stable homotopy group $\left\{S^{0}, F^{+}\right\}$as follows. Here $X^{+}$ means $X$ union with a disjoint base point. 


\section{BECKER - GOTTLIEB}

First we embed $F$ in $\mathrm{R}^{N}$ with the normal bundle $\nu$. We alter $V$, if need be, by multiplication by a positive function $k(m)$ so that for $m \in \dot{F}$, the vector $k(m) V(m)$ has length greater than or equal to 1 . Then define $\tau_{V}$ as the composition

$$
\tau_{V}: S^{N} \stackrel{c}{\longrightarrow} F^{\nu} / \dot{F}^{\nu} \stackrel{i_{V}}{\longrightarrow} F^{\tau \oplus \nu}=S^{N} \wedge F^{+}
$$

Here $F^{\nu}$ is the Thom space of $\nu$; that is the total space of $\nu$ with the subspace of vectors of length greater than or equal to 1 identified to a point. Now $F$ is embedded inside of $S^{N}$ with tubular neighborhood $\nu$. Then $c$ is the collapsing map. Now $\tau$ denotes the tangent bundle of $F$, so $\tau \oplus \nu$ is a trivial bundle, hence the Thom space is $S^{N} \wedge F^{+}$. Finally $i_{V}$ is the map defined by $i_{V}\left(\vec{v}_{m}\right)=\left(V(m), \vec{v}_{m}\right)$ where $\vec{v}_{m} \in \nu$. Since $\|V(m)\| \geq 1$ for $m \in \dot{F}$, we see that $i_{V}$ takes $\dot{F}^{\nu}$ to the base point of $F^{\tau \oplus v}$.

\subsection{Properties of $\tau_{V}$}

a) $\left\{\tau_{V}\right\}$ is independent of the embedding of $F$ in $\mathrm{R}^{N}$ and independent of the $N$ in $\mathrm{R}^{N}$.

b) $\left\{\tau_{V}\right\}=\left\{\tau_{V^{\prime}}\right\}$ if $V$ is homotopic to $V^{\prime}$ by a proper homotopy of vector fields. That is $V_{t}$ is a homotopy such that no zero occurs on $\dot{F}$ during the homotopy.

c) $\left\{\tau_{-V}\right\}=(-1)^{n}\left\{\tau_{V}\right\}$ where $n=\operatorname{dim} F$.

d) $\left\{\tau_{V}\right\}=0$ if $V$ has no zeros.

e) Let $V \times W$ be a vector field on $A \times B$. Then $\tau_{V \times W}=\tau_{V} \wedge \tau_{W}$. Proof. c) This follows since $\tau_{-V}=r \circ \tau_{V}$ where $r: \tau \oplus \nu \rightarrow \tau \oplus \nu$ sends $(u, v) \mapsto(-u, v)$. This gives rise to a map $S^{N} \wedge F^{+} \rightarrow$ $S^{N} \wedge F^{+}$which changes the sign of the first $n$ coordinates of $S^{N}=$ $S^{n} \wedge S^{N-n}$.

e) It is easy to check that $\tau_{V \times W}$ is represented by

$$
\begin{aligned}
S^{2 N} & \rightarrow S^{N} \wedge S^{N} \rightarrow\left(A^{\nu} / \dot{A}^{\nu}\right) \wedge\left(B^{\mu} / \dot{B}^{\mu}\right) \\
& \stackrel{i_{V} \wedge i_{W}}{\longrightarrow} A^{\tau \oplus \nu} \wedge B^{\tau \oplus \mu}=S^{2 N} \wedge(A \times B)^{+}
\end{aligned}
$$




\section{BECKER - GOTTLIEB}

so $\tau_{V \times W}=\tau_{V} \wedge \tau_{W}$.

\subsection{Definition of Index}

Let $p: S^{N} \wedge F^{+} \rightarrow S^{N}$ be induced by the projection onto the first factor. Then we have $p \circ \tau_{V}: S^{N} \rightarrow S^{N}$. Define the index of $V$ to be

$$
\text { Ind } V=\operatorname{deg}\left(p \circ \tau_{V}\right) .
$$

\subsection{Properties of index}

a) Index is well defined.

b) Index is invariant under proper homotopy.

c) $\operatorname{Ind}(-V)=(-1)^{n} \operatorname{Ind}(V)$.

d) If $V$ has no zeros, then $\operatorname{Ind}(V)=0$.

e) If $V \times W$ is a vector field on $A \times B$ then

$$
\operatorname{Ind}(V \times W)=(\operatorname{Ind} V)(\operatorname{Ind} W) .
$$

Proof. e) $p \circ \tau_{V \times W}=\left(p_{1} \wedge p_{2}\right) \circ\left(\tau_{V} \wedge \tau_{W}\right)=\left(p_{1} \circ \tau_{V}\right) \wedge\left(p_{2} \circ \tau_{W}\right)$ so $\operatorname{deg}\left(p \circ \tau_{V \times W}\right)=\operatorname{deg}\left(p \circ \tau_{V}\right) \cdot \operatorname{deg}\left(p \circ \tau_{W}\right)$.

\section{The index of a local vector field}

Now we extend our definitions of $\tau_{V}$ and Ind $V$ for $V$ which are defined locally on open subsets of $F$. This leads to the index of such vector fields in the same manner as above.

Definition. A vector field $V$ defined on an open set $U$ of $F$ is proper if $\|V(m)\| \geq a>0$ for some $a$ and all $m \in U-C$ where $C$ is some compact subset of $F$. (The reason for this definition is that we can multiply $V$ by a function $k(m)>0$ so that $\|k(m) V(m)\| \geq$ 1 as $m$ approaches the frontier of $U$ ).

Definition An otopy from $V$ to $V^{\prime}$ is a vector field $W$ on $F \times I$ such that $W$ is vector field defined on an open set of $F \times I$ so that every vector $W(m, t)$ is tangent to $F \times t$ and $W(m, 0)=V(m)$ and $W(m, 1)=V^{\prime}(m)$. The otopy is proper if $W$ is a proper vector field. 


\section{BECKER - GOTTLIEB}

\subsection{Definition of $\tau_{V}$}

$$
\tau_{V}: S^{N} \stackrel{c}{\longrightarrow} U^{\nu} \stackrel{i_{V}}{\longrightarrow} F^{\tau \oplus \nu}=S^{N} \wedge F^{+}
$$

where $c$ collapses $S^{N}$ to $F^{\nu} / \dot{F}^{\nu}$ and then collapses every point outside of $U^{\nu}$ to a point, and $i_{V}: \vec{v}_{m} \mapsto\left(V_{m}, \vec{v}_{m}\right)$. Note $i_{V}$ is well defined since $\|V(m)\| \geq 1$ near the frontier of $U$. Here $U^{\nu}$ is the one point compactification of $\nu \mid U$.

\subsection{Properties of $\tau_{V}$}

As before, we obtain the same properties for $\tau_{V}$ for local proper vector fields.

a) $\left\{\tau_{V}\right\}$ is well-defined, that is independent of choices of embedding.

b) $\left\{\tau_{V}\right\}=\left\{\tau_{V^{\prime}}\right\}$ if $V$ is properly otopic to $V^{\prime}$.

c) $\left\{\tau_{-V}\right\}=(-1)^{n}\left\{\tau_{V}\right\}$.

d) $\left\{\tau_{V}\right\}=0$ if $V$ has no zeros.

e) $\tau_{V \times W}=\tau_{V} \wedge \tau_{W}$.

f) If $W=V$ restricted to some open set and if $V$ and $W$ are proper and $W$ has no additional zeros then $\left\{\tau_{W}\right\}=\left\{\tau_{V}\right\}$.

g) If $U$ and $V$ are proper local vector fields defined on disjoint open subsets, then $\left\{\tau_{W}\right\}=\left\{\tau_{U}\right\}+\left\{\tau_{V}\right\}$ where $W$ is the vector field consisting of $U$ and $V$.

Proof. b) Observe that a proper otopy between $V$ and $V^{\prime}$ gives rise to a homotopy from $\tau_{V}$ to $\tau_{V^{\prime}}$.

g)

$$
\tau_{W}: S^{N} \rightarrow F^{\nu} / \dot{F}^{\nu} \rightarrow O_{V}^{\nu} \vee O_{V}^{\nu} \stackrel{i_{U} \vee i_{V}}{\longrightarrow} F^{\tau \oplus \nu}=S^{N} \wedge F^{+}
$$

and

$$
\tau_{U}+\tau_{V}: S^{N} \rightarrow S^{N} \vee S^{N} \rightarrow O_{U}^{\nu} \vee O_{V}^{\nu} \stackrel{i_{U} \vee i_{V}}{\longrightarrow} F^{\tau \oplus \nu}=S^{N} \wedge F^{+}
$$




\section{BECKER - GOTTLIEB}

where $O_{U}^{\nu}$ is the open set of definition of $U$ and $O_{V}^{\nu}$ is the open set for $V$.

\subsection{Definition of Ind $V$}

The index of a local proper vector field is defined to be, as before, $\operatorname{deg}\left(p \circ \tau_{V}\right)$.

\subsection{Properties of Ind $V$}

a) $\operatorname{Ind}(V)$ is well-defined.

b) $\operatorname{Ind}(V)$ is invariant under proper otopy. (In fact the following can be shown: Let $M$ be connected. Then $V$ is properly otopic to $W$ if and only if $\operatorname{Ind}(V)=\operatorname{Ind}(W)$. This in turn implies for global vector fields on a compact manifold with connected nonempty boundary, that $V$ is properly homotopic to $W$ if and only if $\operatorname{Ind}(V)=\operatorname{Ind}(W)$.In both cases there is a vector field whose index equals any arbitrary integer).

c) $\operatorname{Ind}(-V)=(-1)^{n}$ Ind $V$.

d) If $V$ has no zeros then Ind $V=0$.

e) If $V$ and $W$ are proper local vector fields on $A \times B$, then $\operatorname{Ind}(V \times W)=\operatorname{Ind}(V) \cdot \operatorname{Ind}(W)$.

f) Ind $W=$ Ind $V$ if $W$ extends $V$ and has no additional zeros.

g) Ind $W=\operatorname{Ind} U+\operatorname{Ind} V$ if $W$ is defined on two disjoint open sets on which it restricts to $U$ and $V$.

Remark. We also define $\tau_{V}$ for $V$ a vector field defined on a compact submanifold $M$ of $F$, with codimension 0 . Here $\tau_{V}$ is the composition

$$
S^{N} \stackrel{c}{\longrightarrow} F^{\nu} / \dot{F}^{\nu} \rightarrow M^{\nu} / \dot{M}^{\nu} \stackrel{i_{V}}{\longrightarrow} F^{\tau \oplus \nu}=S^{N} \wedge F
$$

All the above properties hold for this $\tau_{V}$. Only a small modification of the definition of proper otopy is necessary. 


\section{BECKER - GOTTLIEB}

\section{The law of vector fields}

Suppose that $M^{k}$ is a submanifold of $F^{n}$ with normal bundle $\gamma$. Then $\tau_{F} \mid M=\tau_{M} \oplus \gamma$. Suppose that $V^{\prime}$ is a vector field on $M$ such that $\left\|V^{\prime}(m)\right\| \geq 1$ for $m \in \dot{M}$. Define the vector field $W$ on the tubular neighborhood $N$ corresponding to $\gamma$ in $F$ by $W\left(\vec{v}_{m}\right)=V^{\prime}(m)+\vec{v}_{m}$. Let $i: M \rightarrow F$ be the inclusion

Lemma 4.1. $\left\{\tau_{W}\right\}=\left\{i \circ \tau_{V^{\prime}}\right\}$.

Proof.

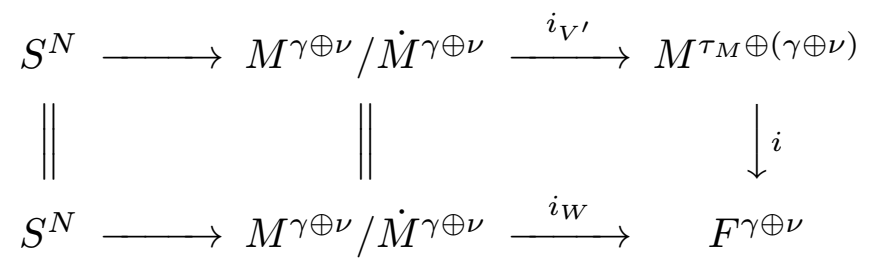

This diagram commutes.

Suppose $F$ is a smooth manifold with a proper vector field $V$. Let $\bar{F}$ be $F$ union with a collar $C=\dot{F} \times I$ and let $V^{\prime}$ be the vector field on $C$ given by $V^{\prime}(m, t)=V(m)+t k \bar{N}(m)$ where $k$ is a constant large enough so that at $\partial \bar{F}=\dot{F} \times\{1\}$ the vector field $V^{\prime}$ points outside. Let $\bar{V}$ be the vector field on $\bar{F}$ which restricts to $V^{\prime}$ on $C$ and to $V$ on $F$.

Lemma 4.2. $\tau_{\bar{V}}=\tau_{V}+\tau_{V^{\prime}}$.

Proof. Apply $3.2 \mathrm{~g}$ ) for compact manifolds to $\bar{F}$ and $\bar{V}$.

Corollary 4.3. Ind $\bar{V}=\operatorname{Ind} V+\operatorname{Ind} V^{\prime}$.

Proof. Compose the right and left sides of the equation in Lemma 4.2 with $p$ and use the definition of index.

We have from Hopf's theorem, since $\bar{V}$ is pointing outside on $\partial \bar{F}$, and the fact that $F$ is homeomorphic to $\bar{F}$, the equation

$$
\text { Ind } \bar{V}=\chi(\bar{F})=\chi(F) \text {. }
$$

Definition. Let $\partial_{-} V$ to be a vector field on $\dot{F}$ defined on the open set $\partial_{-} \dot{F} \subset \dot{F}$ where $\partial_{-} \dot{F}$ consists of those points in $\dot{F}$ such 


\section{BECKER - GOTTLIEB}

that $V(m)$ points inside. Define $\partial_{-} V(m)$ to be projection of $V(m)$ tangent to $F$ for all $m \in \partial_{-} F$. Thus $\partial_{-} V$ is the end of the sequence

$$
V \rightarrow V \mid \partial_{-} F \stackrel{\text { Proj }}{\longrightarrow} \partial_{-} V .
$$

Then $\tau_{\partial_{-} V}: S^{N} \rightarrow S^{N} \wedge \dot{F}^{+}$.

Lemma 4.4. Let $i: \dot{F} \rightarrow F$. Then $i \circ \tau_{\partial_{-} V}=\tau_{V^{\prime}}$.

Proof. This follows from Lemma 4.1. Here $\dot{F}=M$ and its boundary is empty. We can homotopy $V^{\prime}$ by multliplying by a positive function so that the new $V^{\prime}$ when restricted to $\partial_{-} F \times \frac{1}{2}$ is tangent to $\partial_{-} F$ and equal to $\partial_{-} V$ and in a product neighborhood of $\partial_{-} F \times \frac{1}{2}$ the altered $V^{\prime}$ looks like the normal vector field crossed with $\partial_{-} V$.

Theorem 4.5. $\tau_{\bar{V}}=\tau_{V}+i \tau_{\partial-V}$.

Corollary 4.6 The Law of Vector Fields $\chi(F)=\operatorname{Ind} V+\operatorname{Ind} \partial_{-} V$.

Proof. Compose the above equation with Projection.

\section{Construction of the global vector field transfer}

We assume that $F$ is a smooth compact manifold with boundary $\dot{F}$. We assume that $F \stackrel{i}{\longrightarrow} E \stackrel{\pi}{\longrightarrow} B$ is a fibre bundle so that the vector bundle of tangents along the fibre, denoted by $\alpha$, can be defined. In particular, this occurs when $B$ is a smooth manifold and $\pi$ is a smooth map, so $E$ itself is a smooth manifold. Another case when $\alpha$ exists is when the structure group of the bundle is $\operatorname{Diff}(F)$ and $B$ is a finite CW- complex.

Suppose there exists an embedding

$$
j: E \rightarrow \mathrm{R}^{N} \times B
$$

with normal bundle $\nu$. The embedding is assumed to be such that (Proj) $\circ j=\pi$ where Proj : $\mathrm{R}^{N} \times B \rightarrow B$ is projection on the second factor.

We can construct such an embedding in the case when $E$ is a smooth manifold by first embedding $E$ smoothly into $\mathrm{R}^{N}$ for some 


\section{BECKER - GOTTLIEB}

$\mathrm{R}^{N}$. Denote this embedding by $j^{\prime}: E \rightarrow \mathrm{R}^{N}$. Then the required $j$ is given by $j(e)=\left(j^{\prime}(e), p(e)\right)$.

Now let $\dot{E}=\{e \in E \mid e$ is in the boundary of some fibre $\}$. Then $\dot{E}$ is a subspace of $E$ and the restriction of $\pi: E \rightarrow B$ gives a fibre bundle $\dot{F} \rightarrow \dot{E} \rightarrow B$.

Now we say that $V$ is a vector field along the fibres of $E$ if $V$ is a cross-section from $E$ to the total space of $\alpha$, the bundle of tangents along the fibre. We say $V$ is a proper vector field if $V$ has no zeros on $\dot{E}$. For short, we say that $V$ is a vertical vector field if it is a proper vector field along the fibres.

We define $i_{V}: \nu \rightarrow \alpha \oplus \nu$ to be the vector bundle map $\vec{v}_{e} \mapsto$ $\left(V(e)+\vec{v}_{e}\right)$ where $\vec{v}_{e}$ is a vector in $\nu$ which projects down onto $e \in E$. Then we extend $i_{V}$ to a map of the Thom spaces $i_{V}: E^{\nu} \rightarrow$ $E^{\alpha \oplus \nu}$. Now if $E^{\nu}$ is constructed by identifying any vector $\vec{v}_{e} \in \nu$ of length $\left\|\vec{v}_{e}\right\| \geq 1$ to a point, we may regard $i_{V}: E^{\nu} / \dot{E}^{\nu} \rightarrow E^{\alpha \oplus \beta}$ as a map with domain $E^{\nu} / \dot{E}^{\nu}$. We may do this since $V$ restricted to $E$ is nonzero and we may vertically homotopy $V$ until every vector of $V$ on $E$ has length $\geq 1$, so then $\dot{E}^{\nu}$ is sent to a point and so $i_{V}$ can be pushed down to the quotient.

We let $\hat{\pi}:\left(\mathrm{R}^{N} \times B\right)^{+} \rightarrow E^{\nu} / \dot{E}^{\nu}$ be the collapsing map onto the tubular neighborhood of $E$ in $\mathrm{R}^{N} \times B$, followed by identifying $\dot{E}^{\nu}$ to a point. Here $B^{+}$denotes the disjoint union of $B$ and a base point + .

\subsection{Definition of $\tau_{V}$}

The transfer $\tau_{V}$ is the $S$-map defined by the following composition.

$$
\tau_{V}: S^{N} \wedge B^{+}=\left(\mathrm{R}^{N} \times B\right)^{+} \stackrel{\hat{\pi}}{\longrightarrow} E^{\nu} / \dot{E}^{\nu} \stackrel{i_{V}}{\longrightarrow} E^{\alpha \oplus \nu}=S^{N} \wedge E^{+}
$$

The last equality follows since $\alpha \oplus \nu$ is a trivial bundle.

\subsection{Properties of $\tau_{V}$.}

a) $\left\{\tau_{V}\right\} \in\left\{B^{+}, E^{+}\right\}$, the stable homotopy class of $\tau_{V}$, is independent of the choice of embeddings and homotopies used in the construction. 


\section{BECKER - GOTTLIEB}

b) $\left\{\tau_{V}\right\}=\left\{\tau_{V^{\prime}}\right\}$ if $V$ is vertically homotopic to $V^{\prime}$.

c) If $B^{\prime} \subset B$ and $E^{\prime}=\pi^{-1}\left(B^{\prime}\right)$ and $V^{\prime}=V \mid E^{\prime}$ and $j: E^{\prime} \rightarrow E$ and $i: B^{\prime} \rightarrow B$ are inclusions, then $j \circ \tau_{V^{\prime}}=\tau_{V} \circ i$.

d) The fibre bundle $F \rightarrow X \times E \stackrel{1 \times \pi}{\longrightarrow} X \times B$ with vertical vector field $W=0 \times V$ has a transfer $\tau_{W}=1 \wedge \tau_{V}$. This follows from

$$
\begin{array}{r}
\tau_{W}: S^{N} \wedge X \wedge B^{+}=S^{N} \wedge(X \times B)^{+}=\left(\mathrm{R}^{N} \times X \times B\right)^{+} \rightarrow \\
(X \times E)^{\text {Proj }^{*} \nu} /(X \times \dot{E})^{\text {Proj }^{*} \nu} \rightarrow(X \times E)^{\left(\text {Proj }^{*} \alpha\right) \oplus\left(\text { Proj }^{*} \nu\right)} \\
=S^{N} \wedge(X \times E)^{+}=S^{N} \wedge X \wedge E^{+}
\end{array}
$$

e) The following diagram commutes (where $\Delta$ is the diagonal map).

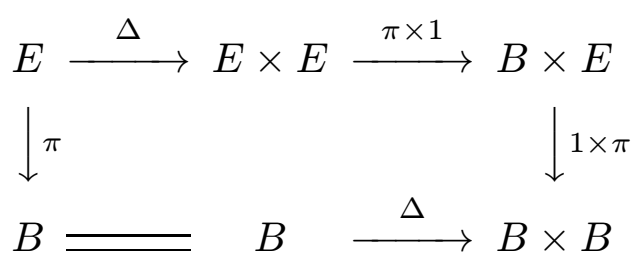

This gives rise to the commuting diagram

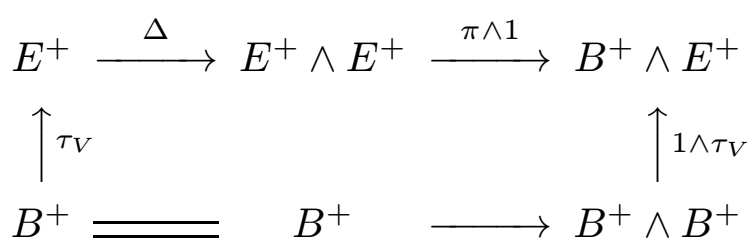

Thus for cohomology theories with cup products,

$$
\tau_{V}^{*}\left(\pi^{*}(x) \cup y\right)=x \cup \tau_{V}^{*}(y)
$$

\section{Local vector field transfers for fibre bundles}

Suppose that $M$ is a smooth submanifold of codimension 0 contained in $E$. Suppose $W$ is a vertical vector field defined on 


\section{BECKER - GOTTLIEB}

$M \subset E$ such that $W \mid \partial M$ consists of vectors of length greater than or equal to 1 .

6.1. Definition of $\tau_{V}$

$$
\begin{aligned}
\tau_{V}: S^{N} \wedge B^{+} & \stackrel{\hat{\pi}}{\longrightarrow} E^{\nu} / \dot{E}^{\nu} \stackrel{c}{\longrightarrow} M^{\nu} / \dot{M}^{\nu} \stackrel{i_{V}}{\longrightarrow} M^{\alpha \oplus \mu} \\
& =S^{N} \wedge M^{+} \stackrel{\Sigma j}{\longrightarrow} S^{N} \wedge E^{+}
\end{aligned}
$$

where $c$ collapses $E$ to $M$ and $\Sigma j$ is the suspension of the inclusion $M \stackrel{j}{\hookrightarrow} E$.

\subsection{Properties of $\tau_{V}$}

The locally defined transfer for vertical vector fields satisfies analogous properties possessed by the globally defined transfer

a) $\left\{\tau_{V}\right\} \in\left\{B^{+}, E^{+}\right\}$is independent of the choice of embedding and homotopies in the construction of $\tau_{V}$

b) $\left\{\tau_{V}\right\}=\left\{\tau_{V^{\prime}}\right\}$ if $V$ is vertically otopic to $V^{\prime}$.

c) If $B^{\prime} \subset B$ and $E^{\prime}=\pi^{-1}\left(B^{\prime}\right)$ and $V^{\prime}=V \mid M \cap E^{\prime}$ and $M \cap E^{\prime}$ is a codimension 0 manifold in $E^{\prime}$, then $j \circ \tau_{V^{\prime}}=\tau_{V} \circ i$ where $i$ and $j$ are the appropriate inclusions.

d) Analogues of Properties d) and e) of section 5.2 hold. In particular $\tau_{V}^{*}\left(\pi^{*}(x) \cup y\right)=x \cup \tau_{V}^{*}(y)$ holds.

e) If $V$ is a local vertical vector field defined on disjoint codimension 0 submanifolds $M_{1}$ and $M_{2}$ so that $\|V(m)\| \geq 1$ when $m \in \partial\left(M_{1} \cup M_{2}\right)$, then $\left\{\tau_{V}\right\}=\left\{\tau_{V_{1}}\right\}+\left\{\tau_{V_{2}}\right\}$ where $V_{1}=V \mid M_{1}$ and $V_{2}=V \mid M_{2}$.

Proof. e)

$$
\tau_{V}: S^{N} \wedge B^{+} \rightarrow\left(M_{1}^{\nu} / \dot{M}_{1}^{\nu}\right) \vee\left(M_{2}^{\nu} / \dot{M}_{2}^{\nu}\right) \rightarrow S^{N} \wedge E^{+}
$$

Theorem 6.1. $\tau_{V}^{*} \circ \pi^{*}: H^{*}(B) \rightarrow H^{*}(B)$ is multiplication by $\operatorname{Ind}(V \mid F)$,

Proof. Let $V$ be a proper vector field defined on an open set of $F$. Then $\tau_{V}: F^{+} \rightarrow S^{0}$ can be regarded as the transfer of the trivial fibre bundle $F \stackrel{\pi^{\prime}}{\longrightarrow} *$. Now $\tau_{V} \pi^{*}=\operatorname{Ind}(V)$. Now suppose that $W$ 


\section{BECKER - GOTTLIEB}

is a vertical vector field on $F \rightarrow E \stackrel{\pi}{\longrightarrow} B$ so that $W \mid F=V$. Then by property c), $\tau_{V}^{*} \circ j^{*}=i^{*} \circ \tau_{W}^{*}$. Hence

$$
\tau_{V}^{*} \circ j^{*} \circ \pi^{*}(1)=i^{*} \circ \tau_{W} \circ \pi^{*}(1)
$$

so Ind $V=\tau^{*} \circ \pi^{\prime *}(1)=\tau_{V}^{*} \circ j^{*} \circ \pi^{*}(1)=i^{*}\left(\tau_{W}^{*}(1)\right)$ so $\tau_{W}^{*}(1)=$ Ind $V \in H^{0}(B)$. Now from d), $\tau_{W}^{*}\left(\pi^{*}(x)\right)=\tau_{W}^{*}\left(\pi^{*}(x) \cup 1\right)=$ (Ind $V) x$.

\section{Transfer formula}

Let $F \rightarrow E \stackrel{\pi}{\longrightarrow} B$ be a vector bundle with $F$ a compact manifold and $V$ a vertical vector field on $E$. Then $\dot{E} \stackrel{\pi}{\longrightarrow} B$ is a subbundle with fibre $\dot{F}$ and inclusion map $i: \dot{E} \rightarrow E$. There is a a vector field $\partial_{-} V$ on $\dot{E}$ formed by taking those vectors of $V$ on $\dot{E}$ which point inside $E$ and projecting them down so that they are tangent to $\dot{E}$. Thus $\partial_{-} V$ is a vertical vector field defined on an open set of $\dot{E}$. We choose a manifold with boundary $M \subset \dot{E}$ which contains all the zeros of $\partial_{-} V$. Then we define a transfer $\tau_{\partial_{-} V}: B^{+} \rightarrow \dot{E}^{+}$.

Theorem 7.1. $\tau=\tau_{V}+i \tau_{\partial_{-} V}$ where $\tau$ is the usual Euler-Poincare transfer.

Corollary $7.2 \chi(F)=$ Ind $V_{1}+$ Ind $\partial_{-} V_{1}$ where $V_{1}$ is the restriction of $V$ to the fibre $F$.

Proof. Consider $\tau^{*} \circ \pi^{*}$ in singular cohomology. Then

$$
\tau^{*} \circ \pi^{*}=\tau_{V}^{*} \circ \pi^{*}+\tau_{\partial_{-} V}^{*} \circ i^{*} \circ \pi^{*}
$$

Now the composition of transfers with projections is multiplication by the index of the appropriate vector field restricted to the fibre. So the corollary is true. Note that this is just the vector field law for the vector field restricted to the fibre $F$.

To prove the theorem we must add a collar $C$ onto $\dot{E}$ and then extend $V$ to $\bar{V}$ over the collar so that at the new boundary $\bar{V}$ points outside. 


\section{BECKER - GOTTLIEB}

Let $V$ be a vertical vector field on the fibre bundle $F \stackrel{i}{\longrightarrow} E \stackrel{\pi}{\longrightarrow}$ $B$. Here $F$ is a compact smooth manifold. Let $\vec{N}$ be a unit normal outward pointing vertical vector field defined on $\dot{E}$. Let $C=\dot{E} \times I$ be a collar for $E$ so that $C=\dot{E} \times I \rightarrow B$ is a fibre bundle with fibre $\partial F \times I$. Extend $V$ over $C$ by $V^{\prime}(e, t)=V(e)+t k \vec{N}$ where $k$ is a constant so large that $V(e, 1)$ is pointing outside. Let $\bar{V}$ be the vertical vector field on $E \cup C$ so that $\bar{V} \mid E=V$ and $\bar{V} \mid C=V^{\prime}$. Then by Section 2 and Section 3 we see that

$$
\left\{\tau_{\bar{V}}\right\}=\left\{\tau_{V}\right\}+\left\{\tau_{V^{\prime}}\right\}
$$

Now $\tau_{\bar{V}}$ is the usual Euler-Poincare transfer, and $E \stackrel{\pi}{\longrightarrow} B$ and $E \cup C \rightarrow B$ are fibre bundle equivalent, so we can identify $\tau_{V}$ for $E \stackrel{\pi}{\longrightarrow} B$ with the $\tau_{V}$ for $E \cup C \rightarrow B$. Now $\tau_{V^{\prime}}$ can be thought of as $j \tau_{V^{\prime}}$ where $\tau_{V^{\prime}}: B^{+} \rightarrow C^{+}$and $j: C \rightarrow E$. Now $\left\{\tau_{V^{\prime}}\right\}=\left\{\tau_{V^{\prime \prime}}\right\}$ where $V^{\prime \prime}=V \mid\left(\partial_{-} E\right) \times I$ where $\partial_{-} E$ is the open set of $\dot{E}$ where $V$ points inwards. We may adjust the definition of $V^{\prime \prime}$ so that $V^{\prime \prime} \mid\left(\partial_{-} E \times \frac{1}{2}\right)$ is the same as $V$ projected down onto $\partial_{-} E$. So locally near $\partial_{-} E \times \frac{1}{2}$ we see that $V^{\prime \prime}$ can be vertically homotopied to $\left(\partial_{-} V\right) \times A$ where $A$ is the vector field on $I$ with the zero at $\frac{1}{2}$ and always pointing outside. So $\left\{\tau_{V^{\prime \prime}}\right\}=\left\{i \tau_{\partial_{-} V}\right\}$.

\section{Equivariant vector fields}

One way to construct vertical vector fields is to first look for equivariant vector fields. Let $G$ act on $F$ smoothly, so that $G$ also acts on the tangent space $T(F)$. Then $V$ is an equivariant vector field on $F$ if $g_{*}(V(m))=V(g(m))$. Now we define the universal fibration with fibre $F$ and group $G$ by

$$
F \rightarrow E_{G} \times_{G} F \stackrel{p}{\longrightarrow} B_{G}
$$

Then the equivariant $V$ give rise to a vector field along the fibres $\bar{V}$ as follows. Let $T(F)$ be the total space of the tangent bundle of $F$. Then $G$ acts on $T(F)$ and $p: T(F) \rightarrow F$ is a $G$-map. 


\section{BECKER - GOTTLIEB}

Since $V$ is equivariant, we may think of $V$ as a $G$-cross-section $F \rightarrow T(F)$. So we have

$$
\alpha=E \times_{G} T(F) \stackrel{1 \times_{G} p}{\underset{V}{\rightleftarrows}} E_{G} \times F .
$$

where $1 \times_{G} p:\left\langle e, \vec{v}_{m}\right\rangle_{G} \mapsto\langle e, m\rangle_{G}$ and $\bar{V}:\langle e, m\rangle_{G} \rightarrow\langle e, V(m)\rangle_{G}$. That is $\bar{V}$ is a cross-section to $1 \times_{G} p$, so $\bar{V}$ is a vector field along the fibres. Note that $\bar{V} \mid F=V$. If $V$ is proper, then $\bar{V}$ is a vertical vector field. This $\bar{V}$ pulls back to a vertical vector field $W$ on induced bundles. So on each of those pullbacks which in addition admit a bundle $\nu$ so that $\alpha \oplus \nu$ is trivial, there is a transfer $\tau_{W}$. Then $p_{*} \circ \tau_{W}$ is multiplication by $\operatorname{Ind}(W \mid F)$. Hence we have the following theorem, where $\operatorname{tr}(G, F)$ is the trace of the action of $G$ on $F$ defined in [3].

Theorem $8.1 \operatorname{tr}(G, F) \mid$ Ind $V$.

Corollary $\mathbf{8 . 2}$ a) If $G$ acts freely on $F$ and $G$ is finite, then

$|G| \mid$ Ind $V$

b) If $G \cong\left(\mathrm{Z}_{p}\right)^{n}$, then the order of the smallest orbit divides Ind $V$.

c) If $G$ is a connected Lie group and Ind $V \neq 0$, then the maximal torus is contained in some isotropy subgroup.

Theorem 8.2 Let $V$ be an equivariant vector field on a compact manifold $F$ with $G$ a compact Lie Group. Then $\tau_{V}=\tau-i_{1} \tau_{1}+$ $i_{2} \tau_{2}-i_{3} \tau_{3}+\ldots$ where $\tau_{j}$ is the Euler-Poincare transfer for some sub-bundle and $i_{j}$ are inclusion maps.

Proof. First we can assume by Mostow's theorem that $F$ has an equivariant Riemannian metric $\langle$,$\rangle . So we have for all g \in G$

$$
\left\langle g_{*} \vec{v}, g_{*} \vec{w}\right\rangle=\langle\vec{v}, \vec{w}\rangle
$$

Now if $d(x, y)$ is the metric arising from the equivariant Riemannian metric $\langle$,$\rangle , we have that d$ is equivariant also. That is, for all $g, x$ and $y$,

$$
d(g(x), g(y))=d(x, y)
$$




\section{BECKER - GOTTLIEB}

Lemma 8.4 If $V$ is an equivariant vector field, then

a) $\partial V$ is equivariant on $\partial F$.

b) The unit normal vector field $\vec{N}$ is equivariant.

Proof. We must show that $g_{*}(\vec{N}(m))$ is a unit vector normal to $\partial F$ at $g(m)$. Now $\partial F$ is a $G$-subspace of $F$, so $g_{*}$ takes vectors tangent to $\partial F$ into vectors tangent to $\partial F$. Hence if $\vec{v}_{m}$ is tangent to $\partial F$ at $m$ then $\left\langle\vec{v}_{m}, \vec{N}(m)\right\rangle=0$. But

$$
0=\left\langle\vec{v}_{m}, \vec{N}(m)\right\rangle=\left\langle g_{*}\left(\vec{v}_{m}\right), g_{*} \vec{N}(m)\right\rangle
$$

so $g_{*}(\vec{N}(m))$ is orthogonal to the image of $g_{*}$ of vectors tangent to $\partial F$ at $m$. Since $g_{*}$ is an isomorphism, $g_{*}(\vec{N}(m))$ is orthogonal to all tangent vectors to $\partial F$ at $g(m)$. Also

$$
\left\langle g_{*} \vec{N}(m), g_{*} \vec{N}(m)\right\rangle=\langle\vec{N}(m), \vec{N}(m)\rangle=1
$$

so $g_{*} \vec{N}(m)$ is a unit vector. Also $g_{*} \vec{N}(m)$ points outside. Hence $g_{*} \vec{N}(m)=\vec{N}(g(m))$ by definition. This proves b). We prove a) by observing that

$$
\vec{V}(m)=\partial \vec{V}(m)+k \vec{N}(m)
$$

for some $k(m)$. Then the fact that $\vec{V}$ and $\vec{N}$ are equivariant implies $\partial \vec{V}$ is equivariant also.

Lemma 8.5. $\partial_{-} F, \partial_{+} F$ and $\partial_{0} F$ are $G$-spaces and the vector fields $\partial_{-} V, \partial_{+} V$ and $\partial_{0} V$ are equivariant.

Proof. This lemma is a corollary of the previous lemma. Note that $k>0$ when $m \in \partial_{+} F$ and $k<0$ when $m \in \partial_{-} F$ and $k=0$ when $m \in \partial_{0} F$. Thus $\partial_{-} F$ is equivariant since $k=\langle\vec{V}, \vec{N}\rangle$. So $\partial_{-} V$ is equivariant also.

Let $D: \partial_{-} F \rightarrow \mathrm{R}$ be given by $D(m)=\left\langle\partial_{-} V(m), \partial_{-} V(m)\right\rangle$. Now $g(D(m))=D(g(m))$. There is an $a \in \mathrm{R}$ not zero such that 


\section{BECKER - GOTTLIEB}

$D^{-1}(a)$ is a closed equivariant manifold, and also $\partial_{-} V \mid D^{-1}([a, \infty])$ has no zeros. Hence

$$
\text { Ind } \partial_{-} V=\operatorname{Ind} \partial_{-} V \mid D^{-1}([0, a)) \text {. }
$$

Now let $\partial_{-} E=E_{G} \times_{G}\left(D^{-1}([a, \infty))\right.$. Then $\partial_{-} E \stackrel{p}{\longrightarrow} B$ is a fibre bundle which is a subbundle of $E \stackrel{p}{\longrightarrow} B$ and $i: \partial_{-} E \rightarrow E$ is fibre preserving and $\partial_{-} V$ leads to a vector field $\partial_{-} \bar{V}$ along the fibre of $\partial_{-} E$. So Theorem 7.1 states that

$\tau_{1}=\tau_{\bar{V}}+i \tau_{\partial_{-} \bar{V}}$ where $\tau_{1}$ is the Euler-Poincare transfer for $E \stackrel{p}{\longrightarrow} B$. So $\tau_{\bar{V}}=\tau_{1}-i \tau_{\partial_{-} \bar{V}}$. Now we do the same thing for $\partial_{-} E \rightarrow B$ by defining $\partial_{--} E$. Then

$$
\tau_{\bar{V}}=\tau_{1}-i\left(\tau_{2}-i_{1} \tau_{\partial_{--} \bar{V}}\right)
$$

We continue this argument until finally $\partial_{-\ldots-} \bar{V}$ is empty.

\section{The evaluation map}

Suppose that $F \rightarrow E \stackrel{p}{\longrightarrow} B$ is a smooth fibre bundle with vertical field $\bar{V}$. Suppose that the bundle of tangents along the fibre $F$ has a finite inverse $\nu$ so that $\alpha \oplus \nu=E \times \mathrm{R}^{N}$ for some $N$. This occurs when $E$ is a compact manifold or when the group $G$ of the fibre bundle acts smoothly on $\mathrm{R}^{N}$ with $F$ a $G$-manifold smoothly imbedded in $\mathrm{R}^{N}$ and the base space $B$ is finite dimensional.

Now we have a transgression map coming from the fibre bundle $\omega: \Omega B \rightarrow F$. Also let $V=\bar{V} \mid F$. Then

Theorem 9.1. (Ind $V) \omega_{*}:\{X, \Omega B\} \rightarrow\{X, F\}$ is trivial for all finite complexes $X$.

Here \{\} denotes the group of stable homotopy classes.

Corollary 9.2. If $G$ is a compact Lie group acting smoothly on $F$ and $\omega: G \rightarrow F$ is the orbit map and $V$ is an equivariant vector field, then (Ind $V)\{\omega\}=0$.

Proof. We consider the fibre bundle $F \rightarrow E_{G} \times_{G} F \rightarrow B_{G}$ with the vertical vector field $\bar{V}$. Then $\omega: \Omega B G \simeq G \stackrel{\omega}{\longrightarrow} F$ can be 


\section{BECKER - GOTTLIEB}

thought of as the transgression or the orbit map. Thus applying $\omega_{*}$ on $1_{G}: G \rightarrow G$ gives (Ind $\left.V\right)\{\omega\}=0$.

Proof of the theorem. Compare [2]. We will show the theorem by considering $X$ as a finite subcomplex of $\Omega B$. Consider the fibre square

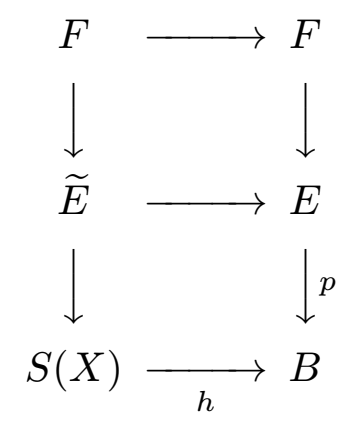

where $\widetilde{E}$ is the pullback of $p$ over the suspension $S(X)$ of $X$ by the map $h$ where $h$ is the adjoint of the inclusion $\bar{h}: X \hookrightarrow \Omega B$. The vector field $\bar{V}$ along the fibre pulls back to a vector field $\widetilde{V}$ along the fibre on $\widetilde{E}$. Consider the diagram

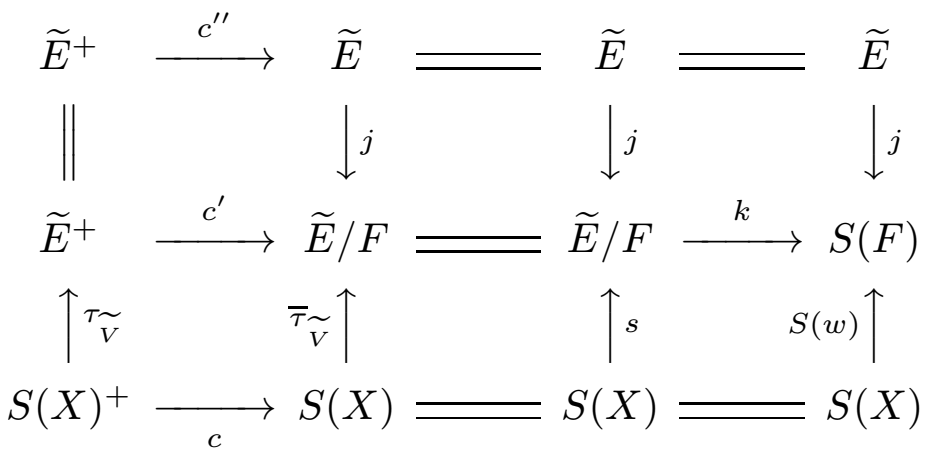

This is a diagram of $S$-maps. The maps $c, c^{\prime}$ and $c^{\prime \prime}$ are the collapsing maps which take the point + onto the base point of the respective targets. The map $j$ is the quotient map. Obviously $c^{\prime}=j c^{\prime \prime}$. So the top left square commutes. Now $\tau_{\widetilde{V}}$ is the transfer associated to $\widetilde{V}$ and $\bar{\tau} \widetilde{V}$ is induced by the transfer on the quotient space, so of course $c^{\prime} \tau_{\widetilde{V}}=\bar{\tau} \widetilde{V} c$. We also denote by $\omega$ the map $X \rightarrow \Omega B \stackrel{\omega}{\longrightarrow} F$. Then $S(\omega)$ is the suspension of $\omega$. Note that $\omega$ 


\section{BECKER - GOTTLIEB}

is involved with the clutching map

$$
\psi: X \times F \rightarrow F
$$

of the fibration $\widetilde{E} \stackrel{p}{\longrightarrow} S(X)$. The evaluation map $\omega$ is the composition

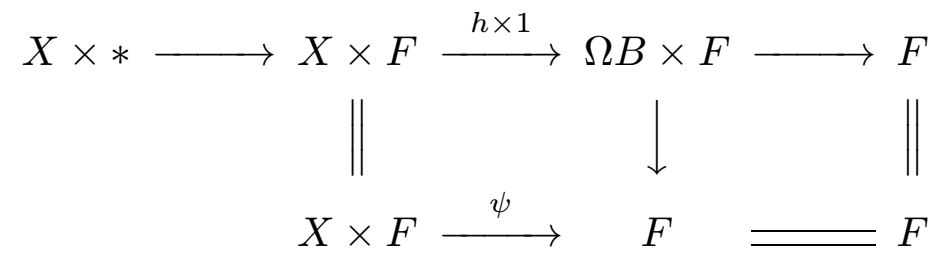

The map $s: S(X) \rightarrow \widetilde{E} / F$ is the cross section defined as follows. $S(X)$-base point is contractible, hence $\widetilde{E} \mid(S(X)$-base point) is fibre homotopy equivalent to the trivial fibration

$$
(S(X)-\text { base point }) \times F
$$

Let $s(b)=b \times *$ where $* \in F$ is the base point of $F$. This extends to $s: S(X) \rightarrow \widetilde{E} / F$.

Now $k: \widetilde{E} / F \rightarrow S(F)$ is the map which appears from the Puppe sequence, namely, the collapsing map of the neighborhood $C X \times F$ of $F$. Then $k j$ is homotopic to a point. Also the left triangle homotopy commutes, that is $S(\omega) \sim k s$.

Lemma 9.3. $\bar{\tau}_{\widetilde{V}}=(\operatorname{Ind} V) s$.

Proof. We may deform $\widetilde{V}$ by a proper homotopy so that $\widetilde{V}$ restricts to $0 \times V$ over $F \times C X$. We can properly homotopy $V$ so that there are a finite number of zeros, each one of them of local index \pm 1 . Then we have as many copies of $s$ as there are zeros of $V$, those $s$ corresponding to +1 zeros represent the same homotopy class, and those associated to the -1 zeros represent the negative of the homotopy class. Hence $\bar{\tau}_{\widetilde{V}}=(\operatorname{Ind} V) s$.

Now we prove the theorem

$$
\text { (Ind } V) S(\omega) c=k \circ(\operatorname{Ind} V) s c=k \bar{\tau}_{\widetilde{V}} c=k c^{\prime} \tau_{\widetilde{V}}=k c^{\prime \prime} j k=*
$$




\section{BECKER - GOTTLIEB}

since $j k$ is homotopic to a constant map. Now $c^{*}:\{S(X), S(F)\} \rightarrow$ $\left\{S(X)^{+}, S(F)\right\}$ given by $c^{*}\{f\}=\{f \circ c\}$ is injective. Hence Ind $V\{S(\omega)\}=0$, proving the theorem.

\section{BIBLIOGRAPHY}

[1] J.C. Becker And D.H. Gottlieb, The transfer map and fiber bundles, Topology, 14 (1975), pp. 1-12.

[2] J.C. Becker and D.H. Gottlieb, Transfer for fibrations and duality, Compositio Mathematica, 33 (1976), pp. 107-133.

[3] D. H. Gottllieb, The trace of an action and the degree of a map, Transactions A.M.S., 293 (1986), pp. 381-410.

[4] A. Dold, Fixed point index and fixed point theorem for Euclidean neighborhood retracts, Topology, 4 (1965), pp. 1-8.

[5] A. Dold, Transfer des points fixes d'une famille continue d'applications, C.R. Acad. Sci. Paris Sér. A, 278 (1974), pp. 1291-1293.

[6] A. Dold, The fixed point index of fibre preserving maps, Inventiones Math., 25 (1974), pp. 215-244.

[7] A. DoLD, The fixed point transfer of fibre-preserving maps, Math. Z., 148 (1976), pp. 215-244.

[8] A. Dold, Fixed point theory and homotopy theory, Contemporary Mathematics, 12 (1982), pp. 105-115.

[9] A. Dold And D. Puppe, Duality trace and transfer, Proceedings of the Steklov Institute of Mathemataics, Issue 4 (1984), pp. 85-103.

[10] Jan Rudzinski, Transfer and its reduction to coverings, Bulletin de l'academie Polanaise des Sciences, serie des sciences mathematique, 27 (1979), pp. 913-921.

James C. Becker

Daniel H. Gottlieb

Department of Mathematics

Purdue University

West Lafayette, IN 47907

U.S.A 